\title{
A Compact Index for Order-Preserving Pattern Matching*
}

\author{
Gianni Decaroli ${ }^{1}$, Travis Gagie ${ }^{2}$, and Giovanni Manzini ${ }^{1,3}$ \\ ${ }^{1}$ Computer Science Institute, University of Eastern Piedmont, Italy \\ ${ }^{2}$ Diego Portales University, Santiago, Chile \\ ${ }^{3}$ IIT-CNR, Pisa, Italy
}

\begin{abstract}
Order-preserving pattern matching was introduced recently but it has already attracted much attention. Given a reference sequence and a pattern, we want to locate all substrings of the reference sequence whose elements have the same relative order as the pattern elements. For this problem we consider the offline version in which we build an index for the reference sequence so that subsequent searches can be completed very efficiently. We propose a space-efficient index that works well in practice despite its lack of good worst-case time bounds. Our solution is based on the new approach of decomposing the indexed sequence into an order component, containing ordering information, and a $\delta$ component, containing information on the absolute values. Experiments show that this approach is viable, faster than the available alternatives, and it is the first one offering simultaneously small space usage and fast retrieval.
\end{abstract}

\section{Introduction}

The problem of Order-Preserving Pattern Matching consists in finding, inside a numerical sequence $T$, all subsequences whose elements are in a given relative order. For example, if the pattern is $P=(1,2,3,4,5)$ we need to find all increasing subsequences of length five; so if $T=(10,20,25,30,31,50,47,49)$ we have a first match starting with the value 10 , a second match starting with the value 20 , and no others.

This problem is a natural generalization of the classic exact matching problem where we search for subsequences whose values are exactly those of the pattern. Order-preserving matching is useful to search for trends in time series like stock market data, biomedical sensor data, meteorological data, etc. In the last few years this problem has received much attention. Not surprisingly, most of the results are generalizations of algorithms and techniques used for exact matching. In [1, 8, 20, 21] the authors propose online solutions inspired by the classical linear time Knuth-Morris-Pratt and Boyer-Moore algorithms [19, Chap. 2]. In [9] the authors consider the offline problem in which $T$ can be preprocessed and propose an index that generalizes the classical Suffix Tree data structure [19, Chap. 5]. Finally in 2, 4, 5, 7, 12, 17, the authors consider approaches based on the concept of filtration and seminumerical matching [19, Chap. 4].

\footnotetext{
${ }^{*}$ A preliminary version of this paper appeared in the Proc. IEEE Data Compression Conference, DCC 2017, Snowbird, UT, USA, 2017 [1].
} 
In this paper we extend to Order-Preserving Matching another well known idea of exact matching: simultaneously compressing and indexing a sequence of values [23. In Section 3 we show how to compactly represent a sequence $T$ so that given a pattern $P$ we can efficiently report all subsequences of $T$ whose elements are in the same relative order as the elements of $P$. Our contribution is based on the new idea of decomposing the sequence $T$ into two components: the order component and the $\delta$ component. Informally, the order component stores the information about the relative order of the elements of $T$ inside a window of a preassigned size, while the $\delta$ component contains the information required for reconstructing $T$ given the order component. The order component is stored into a compressed suffix array while the $\delta$ component is stored using an ad-hoc compression technique.

To search for a pattern we compute its ordering information and then we search for it in the compressed suffix array of the order component. Since the information in the order component is only partial, this search gives us a list of potential candidates which are later verified using the $\delta$ component. In other words, the search in the compressed suffix array is a sort of filtering phase that uses the index to quickly select a set of candidates, discarding all other subsequences in $T$ that certainly do not match.

The overall efficiency of our approach depends on some parameters of the algorithm whose influence will be experimentally analyzed in Section 4. The bottom line is that our index takes roughly the same space as the compressor gzip and can report the orderpreserving occurrences of a pattern an order of magnitude faster than simply scanning the input file. Indeed, in Section 4 we show that our solution is significantly faster than the current fastest online algorithm [3] and the offline algorithm in [5] which is also based on the idea of building a "partial" index to speed up the search.

The complete source code for the index construction and search algorithms are available at the repository https://gitlab.com/manzai/order-preserving-index.

\section{Problem formulation and previous results}

Let $T[1, n]$ denote a sequence of $n=|T|$ numerical values. We write $T[i]$ to denote the $i$-th element and $T[j, k]$ to denote the subsequence $T[j] T[j+1] \cdots T[k]$. Given two sequences $P, Q$, we say that they are order isomorphic, and write $P \approx Q$, if $|P|=|Q|$ and the relative order of $P$ 's and $Q$ 's elements is the same, that is

$$
P[i]<P[j] \Longleftrightarrow Q[i]<Q[j] \quad \text { for } 1 \leq i, j \leq|P| .
$$

Hence $(1,3,4,2)$ is order isomorphic to $(100,200,999,101)$ but not to $(1,3,4,5)$. Given a reference sequence $T[1, n]$ and a pattern $P[1, p]$ the order-preserving pattern matching problem consists in finding all subsequences $T[i+1, i+p]$ such that $T[i+1, i+p] \approx P[1, p]$. In this paper we consider the offline version of the problem in which the sequence $T$ is given in advance and we are allowed to preprocess it in order to speed up subsequent searches.

The first algorithms for the order-preserving pattern matching problem were designed for the online case, where one is allowed to preprocess the pattern but not the text, and inspired by the classical Knuth-Morris-Pratt and Boyer-Moore algorithms [1, 8, 20, 21]. The proposed algorithms have guaranteed linear time worst-case complexity or sublinear time average complexity. However, the best results in practice are obtained by algorithms based on the concept of filtration, in which some sort of "order-preserving" fingerprint is applied 
to the text and the pattern [4, 6, 7, 12. The practical performance of these algorithms can be further improved by exploiting the Single Instruction Multiple Data (SIMD) parallelism now commonly offered by modern processors through the SSE instruction set 2, 3]. Note that all the algorithms based on filtration and/or SIMD are superlinear in the worst case.

For the offline problem, Crochemore et al. [10] showed how, given a sequence $T[1, n]$, in $\mathcal{O}(n \log (n) / \log \log n)$ time we can build an $\mathcal{O}(n \log n)$-bit index such that later, given a pattern $P[1, p]$, we can return the starting positions of all the occ order-preserving matches of $P$ in $T$ in optimal $\mathcal{O}(m+$ occ $)$ time. A more space-economical solution is proposed in [15] consisting of an index taking $\mathcal{O}(n \log \log n)$ extra bits in addition to the text. The one in [15] is the first index for order-preserving matching that uses $o(n \log n)$ bits with guaranteed worst case search bounds. Its weaknesseses are that it can handle only patterns whose length is less than a given bound that is polylogarithmic in $n$, and that it returns the position of only one match (if there is one).

In this paper we are interested in practical approaches that work well in practice even if they do not have competitive worst case bounds on the search cost. In [5] Chhabra et al. show how to speedup search building an FM-index [13, 14 on the binary string expressing whether in the input text each element is smaller or larger than the next one. Our proposal can be seen as a generalization of this work: instead of extracting a binary sequence from $T$ we extract information on the relative order of the elements inside a sliding window of size $q$. In addition, we also compute a $\delta$ component containing the information not stored in the order component. As a result we obtain the first compressed representation of a sequence that is simultaneously an index for order-preserving matching. Both our solution and the one in [5] use the index to quickly select a set of candidates, discarding all other subsequences in $T$ that certainly do not match. However, not only we do use a more "informative" index but we also exploit the $\delta$ component to speed up the verification phase obtaining much better performance in practice.

\section{Data representation and search algorithm}

\subsection{The ordering and delta component}

Given a window size $q>1$ and a sequence $T[1, n]$ we define its order component $T_{o}[1, n]$ as follows. For $i=1, \ldots, n$ let $i_{q}=\max (1, i-q+1)$ and define

$$
T_{o}[i]= \begin{cases}0.5 & \text { if } T[i]<\min T\left[i_{q}, i-1\right] \text { or } i=1 \\ k & \text { if } T[i-k]=\max _{i_{q} \leq j<i}\{T[j] \mid T[j] \leq T[i]\} \text { and } T[i-k]=T[i] \\ k+0.5 & \text { if } T[i-k]=\max _{i_{q} \leq j<i}\{T[j] \mid T[j] \leq T[i]\} \text { and } T[i-k]<T[i]\end{cases}
$$

In other words: if $T[i]$ is the smallest element in $T\left[i_{q}, i\right]$ we set $T_{o}[i]=0.5$; otherwise, if $T[i-k]$ is the immediate predecessor of $T[i]$ in $T\left[i_{q}, i-1\right]$ we set $T_{o}[i]=k$ if $T[i]=T[i-k]$, or $T_{o}[i]=k+0.5$ if $T[i]>T[i-k]$. Note that if $T_{o}[i] \geq 1$ then $T\left[i-\left\lfloor T_{o}[i]\right\rfloor\right]$ is the predecessor of $T[i]$ in $T\left[i_{q}, i-1\right]$. The values of $T_{o}$ belong to the set $\{0.5,1,1.5, \ldots, q-0.5\}$ which has $2 q-1$ elements overall. For example, if $q=4$ for $T=(3,8,3,5,-2,9,6,6)$ it is $T_{o}=(0.5,1.5,2,1.5,0.5,2.5,3.5,1)$.

We call $T_{o}$ the order component for $T$ since it encodes ordering information for $T$ 's elements within a size- $q$ window. Formally $T_{o}$ depends also on $q$ but for simplicity we omit it from the notation. Obviously, if $P \approx Q$ then $P_{o}=Q_{o}$. However, we are interested in 
finding the order-preserving occurrences of $P$ within a longer reference sequence $T$ and we will make use of the following more general result.

Lemma 1. Let $i$ be such that $P[1, p] \approx T[i+1, i+p]$. Then, if $P_{o}[j]$ is whole it is $T_{o}[i+j]=P_{o}[j]$; if $P_{o}[j]$ is fractional it is $T_{o}[i+j]=P_{o}[j]$ for every $j$ such that

$$
2 \leq j \leq p \quad \text { and } \quad\left(j-\left\lfloor T_{o}[i+j]\right\rfloor\right) \geq 1 .
$$

Proof. If $k=P_{o}[j]$ is whole, then by definition $P[j]$ is equal to $P[j-k]$ and different from any character in $P[j-k+1, j-1]$. Since $P[1, p] \approx T[i+1, i+p]$ we must similarly have $T[i+j]=T[i+j-k]$ and $T[i+j]$ different from any character in $T[i+j-k+1, i+j-1]$. Hence $T_{o}[i+j]=k=P_{o}[j]$ as claimed.

Let $P_{o}[j]$ be fractional and such that (3) is satisfied. Let $w=\min (q, i+j)$ and $v=\min (q, j)$. Note that $w$ (resp. $v$ ) is the size of the subsequence which is considered for determining $T_{o}[i+j]$ (resp. $P_{o}[j]$ ). Clearly $w \geq v$.

If $T_{o}[i+j]=0.5$, then $T[i+j]$ is the smallest element in the subsequence $T[i+$ $j-w+1, i+j]$. A fortiori $T[i+j]$ is the smallest element in $T[i+j-v+1, i+j]$. The hypothesis $P[1, p] \approx T[i+1, i+p]$ implies that likewise $P[j]$ must be the smallest element in $P[j-v+1, j]$ so it must be $P_{o}[j]=0.5$. Assume now $T_{o}[i+j] \geq 1$ and let $\ell_{j}=j-\left\lfloor T_{o}[i+j]\right\rfloor$. By construction $T\left[i+\ell_{j}\right]$ is the immediate predecessor of $T[i+j]$ in the subsequence $T[i+j-w+1, i+j]$. The condition $j-\left\lfloor T_{o}[i+j]\right\rfloor \geq 1$ implies $1 \leq \ell_{j}<j$.

In other words, if $T_{o}[i+j]$ is fractional and the distance $\left\lfloor T_{o}[i+j]\right\rfloor$ it points back to $T[i+j]$ 's predecessor in $T\left[i_{q}, i+j-1\right]$ is less $j$, then that predecessor is in $T[i+1, i+j-1]$; therefore, since $T[i+1, i+p] \approx P[1, p]$, the distance back from $P[j]$ to it's predecessor is the same. We conclude that $P_{o}[j]=T_{o}[i+j]$ as claimed.

Corollary 2. If $P[1, p] \approx T[i+1, i+p]$ we must have

$$
T_{o}[i+2, i+p]=x_{2} x_{3} \cdots x_{q-1} P_{o}[q] P_{o}[q+1] \cdots P_{o}[p]
$$

where for $j=2, \ldots, q-1$ it is: $x_{j}=P_{o}[j]$ if $P_{o}[j]$ is whole, and either $x_{j}=P_{o}[j]$ or $x_{j} \geq j$ if $P[j]$ is fractional.

Proof. Since $\left\lfloor T_{o}[\cdot]\right\rfloor \leq q-1$, by the above lemma if $P[1, p] \approx T[i+1, i+p]$ then for every $j, q \leq j \leq p$ it is $T_{o}[i+j]=P_{o}[j]$.

For $j=2,3, \ldots, q-1$ the lemma establishes that $T_{o}[i+j]$ can be different from $P_{o}[j]$ only if $P_{o}[j]$ is fractional and $\left.\left\lfloor T_{o}[i+j]\right\rfloor\right)>j-1$. The latter implies $T_{o}[i+j] \geq 1$ as claimed.

In view of the above corollary, our strategy to solve the order-preserving matching problem is to build a compressed full text index for the sequence $T_{o}$. Then, given a pattern $P[1, p]$ with $p>q$, we compute $P_{o}[1, p]$ and then the set of positions $\left\{i_{1}, i_{2}, \ldots, i_{m}\right\}$ satisfying (4). Clearly, we can have $P[1, p] \approx T[i+1, i+p]$ only if $i \in\left\{i_{1}, i_{2}, \ldots, i_{m}\right\}$. Note that finding $\left\{i_{1}, i_{2}, \ldots, i_{m}\right\}$ will usually require more than one distinct search. For example, if $q=4$ and $P=(3,5,2,6,5,1,5)$, then $P_{o}=(0.5,1.5,0.5,2.5,3,0.5,2)$. Then , the sequences to be sought in $T_{o}$ are

$$
\begin{array}{ll}
(1.5,0.5,2.5,3,0.5,2), & (2.5,0.5,2.5,3,0.5,2), \\
(1.5,3.5,2.5,3,0.5,2), & (2.5,3.5,2.5,3,0.5,2),
\end{array}
$$

Clearly, any condition stated in terms of the ordering component only, like Corollary 2 , can only be a necessary condition for an order-preserving match. Thus, we need to verify 
the candidate positions satisfying Corollary 2 in a verification phase using the actual values of the sequence $T$. Since we are interested in indexing and compressing $T$, instead of simply storing $T$ we introduce a representation that takes advantage of the values in $T_{o}$ that are already stored in the index.

Given $T[1, n]$ and $T_{o}[1, n]$, we define a new sequence $T_{\delta}[1, n]$ as follows. Let $T_{\delta}[1]=T[1]$. For $i=2, \ldots, n$ let $i_{q}=\max (1, i-q+1)$ and:

$$
T_{\delta}[i]= \begin{cases}\min T\left[i_{q}, i-1\right]-T[i] & \text { if } T_{o}[i]=0.5 \\ T[i]-T\left[i-\left\lfloor T_{o}[i]\right\rfloor\right] & \text { if } T_{o}[i] \geq 1 .\end{cases}
$$

Notice that for $i \geq 2, T_{\delta}[i] \geq 0$. Indeed, if $T_{o}[i]=0.5$ then by (2) $T[i]<\min T\left[i_{q}, i-1\right]$. If $T_{o}[i] \geq 1$, since $T\left[i-\left\lfloor T_{o}[i]\right\rfloor\right]$ is the immediate predecessor of $T[i]$ in $T\left[i_{q}, i\right]$, it is $T_{\delta}[i] \geq 0$. We call $T_{\delta}$ the $\delta$ component of $T$. While $T_{o}$ provides information on the ordering of $T$ 's elements, $T_{\delta}$ contains information on their absolute values. It is straightforward to verify that given $T_{o}$ and $T_{\delta}$ we can retrieve $T$ in linear time.

Summing up, our approach to compress and index a sequence $T$ and support orderpreserving pattern matching is the following: select a window size $q$ and build the components $T_{o}$ and $T_{\delta}$. Then build a compressed index for $T_{o}$ and compress $T_{\delta}$. These two components together constitute our index. The above description is quite general and can be realized in different ways. In the following we describe our particular implementation and experimentally measure its effectiveness.

\subsection{Representation of the components $T_{o}$ and $T_{\delta}$}

We represent $T_{o}[1, n]$ using a Compressed Suffix Array (csa) consisting of a Huffmanshaped Wavelet Tree built on the BWT of the sequence $T_{o}$. To this end we use the csa_wt class from the sdsl-lite library [18 (in practice we consider the elements of $T_{0}$ multiplied by two to avoid non-integer values). Given any pattern $p$ the csa can compute in $\mathcal{O}(p)$ time the range of rows $[b, e]$ of the Suffix Array of $T_{o}$ prefixed by $p$. To find the actual position in $T_{o}$ of each occurrence of $p$, the csa also stores the set of Suffix Array entries containing text positions which are multiples of a given block size $B$. Then, for each row $r \in[b, e]$ we move backward in the text using the LF-map until we reach a marked Suffix Array entry from which we can derive the position in $T_{o}$ of the occurrence that prefixes row $r$. The above scheme uses $\mathcal{O}(n+(n / B) \log n)$ bits of space and can find the position of all (exact) occurrences of $p$ in $T_{o}$ in $\mathcal{O}(|p|+B$ occ) time, where occ $=e-b+1$ is the number of occurrences. Clearly, the parameter $B$, chosen when we build the csa, offers a trade-off between space usage and running time.

We do not use an index for $T_{\delta}$. However, during the verification phase we need to extract (decompress) the values in random portions of $T_{\delta}$. For this reason we split $T_{\delta}$ into blocks of size $B$ (i.e., the same size used for the blocks in the csa of $T_{o}$ ) and we compress each block independently. The $k$-th block consists of the subsequence $T_{\delta}[k B+1, k B+B]$, except for the last block which has size $(n \bmod B)$. Additionally, we use a header storing the starting position of each block. Hence, given a block index we can decompress it in $\mathcal{O}(B)$ time.

To compactly represent a block of $T_{\delta}$ we take advantage of the fact that the corresponding values in $T_{o}$ are available during compression and decompression. Recalling the definition of $T_{\delta}[i]$ in (5), we partition the values in $T$ into three classes:

1. those such that $T[i]<\min T\left[i_{q}, i-1\right]$ are called minimal; 
2. those such that $T[i]>\max T\left[i_{q}, i-1\right]$ are called maximal;

3. all other values are called intermediate.

The class of $T[i]$ can be determined by both compressor and decompressor, the latter using $T_{o}[i]$, before it is (de)coded. For each block we define

$$
m=\max \left\{T_{\delta}[i] \mid i \text { is minimal }\right\}, \quad M=\max \left\{T_{\delta}[i] \mid i \text { is maximal }\right\} ;
$$

and we store these two values at the beginning of the block. When we encounter a minimal (resp. maximal) value $T[i]$ we know that the corresponding value $T_{\delta}[i]$ will be in the range $[1, m]$ (resp. $[1, M]$ ). When we encounter an intermediate value $T[i]$ if $T_{o}[i]$ is an integer then we know that $T_{\delta}[i]=0$ and there is nothing to encode. If $T_{o}[i]$ is fractional we know that $T_{\delta}[i]$ will be in the range $\left[1, v-T\left[i-\left\lfloor T_{o}[i]\right\rfloor\right]-1\right]$ where $v$ is the smallest element in $T\left[i_{q}, i-1\right]$ larger than $T\left[i-\left\lfloor T_{o}[i]\right\rfloor\right]$.

Summing up, compressing a block of $T_{\delta}$ amounts to compressing a sequence of nonnegative integers $\ell_{1}, \ell_{2}, \ldots, \ell_{B}$ with the additional information that for each $\ell_{i}$ both encoder and decoder know an upper bound $w_{i} \geq \ell_{i}$. We have tested several compressors for this setting and we got the best results using the log-skewed coder 22. Such an encoder

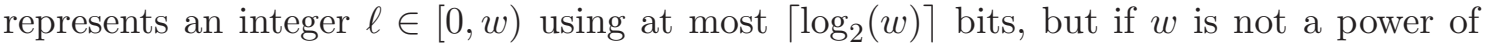
two the smallest values in the range $[0, w)$ are encoded using fewer than $\left\lceil\log _{2}(w)\right\rceil$ bits.

\subsection{Searching for a pattern}

Given the above representations of $T_{o}$ and $T_{\delta}$, we compute the order-preserving occurrences of a pattern $P$ in $T$ as follows. First we compute $P_{o}$ and locate in $T_{o}$ 's csa the row ranges prefixed by each one of the sequences satisfying Corollary 2. If the window size is $q$ there are at most $(q-1)$ ! such sequences. Recall that the basic operation of a csa is the backward search in which, given the range of rows prefixed by a substring $\alpha$ and a character $c$, we find in $\mathcal{O}(1)$ time the range of rows prefixed by $c \alpha$. This suggests we compute the desired set of row ranges with a two-step procedure: first (Phase 1) with $p-q+1$ backward search steps we compute the range of rows prefixed by $P_{o}[q, p]$; then (Phase 2) with additional backward search steps we compute the range of rows prefixed by $x_{2} x_{3} \cdots x_{q-1} P_{o}[q] \cdots P_{o}[p]$ for each $(q-2)$-tuple $x_{2}, \ldots, x_{q-1}$ satisfying the conditions of Corollary 2 , Phase 2 can require up to $q$ ! backward search steps, but the number of steps is also upper bounded by $q$ times the number of row ranges obtained at the end of the phase, which is usually much smaller.

At the end of Phase 2 we are left with a set of rows, each one representing a position in $T$ where an order-preserving match can occur. We verify if there is actually a match (Phase 3) by decompressing the corresponding subsequence of $T$ and comparing it with $P$. Given a row index $r$ representing a position in $T_{o}$ prefixed by a string $x_{2} x_{3} \cdots x_{q-1} P[q] \cdots P[p]$ we use the LF-map to move backwards in $T_{o}$ until we reach a marked position, that is, a position in $T_{o}$ (and hence in $T$ ) which is a multiple of the block size $B$ (say position $\ell B$ ) and marks the beginning of block $\ell$. Each time we apply the LF-map we also obtain a symbol $y_{i}$ of $T_{o}$ hence when we reach the beginning of the block we also have the sequence

$$
y_{1} y_{2} \cdots y_{k} x_{2} x_{3} \cdots x_{q-1} P[q] \cdots P[p]
$$

of $T_{o}$ values from the beginning of the block till the position corresponding to $P[p]$. Using this information and the compressed representation of $T_{\delta}$ (whose blocks can be accessed independently) we retrieve the corresponding $T$ values and determine if there is an actual order-preserving match. 


\begin{tabular}{lrl}
\hline Name & \# Values & Description \\
\hline prices & $31,559,990$ & daily, hourly, and 5min US stock prices \\
temp & $30,505,702$ & max and min daily temperature from 424 US stations \\
ecg & $20,138,750$ & 22 hours and 23 minutes of ECG data \\
rwalk & $50,000,000$ & random walk with integer steps in the range $[-20,20]$ \\
rand & $50,000,000$ & random integers in the range $[-20,20]$ \\
ran127 & $50,000,000$ & random integers in the range $[-127,127]$ \\
\hline
\end{tabular}

Figure 1: Files used in our experiments. All values are 32-bit integers so the size in bytes of the files is four times the number of values.

Phase 3 is usually the most expensive since for each candidate the algorithm has to reach the beginning of the block containing it. We can therefore expect that its running time will be linearly affected by the block size $B$. Note that in our implementation Phase 2 and 3 are interleaved: as soon as we have determined a range of rows prefixed by one of the patterns in Corollary 2 we execute Phase 3 for all rows in the range before considering any other row range.

\section{Experimental results}

Since one of the applications of order-preserving matching is the search of trends in Stock Market data we call our tool "stock market index" (smi from now on). Ours is the first tool combining compression and indexing for the order-preserving matching problem, so we have no direct competitors. We therefore consider separately the compression and search capabilities of our index. We compare the compression ratio achieved by smi with those of gzip and xz: the former has been the standard compression tool for more than 20 years, while the latter is a more recent compressor based on the Lempel-Ziv-Markov chain algorithm (LZMA) that uses more resources but achieves a significantly better compression.

We compare smi's search speed with those of three different tools. As a baseline we used scan, a naive algorithm that simply tries to match the pattern in every text position using the verification algorithm outlined in [7, Sec. 3]. Then, we tested a prototype of the algorithm fm-oppm from [5] that is similar to our approach in that it builds an index to speedup the search. fm-oppm builds an FM-index of the binary string obtained by replacing each text character with 0 or 1 according to whether the next character is smaller than the current one or not. To search for a pattern fm-oppm applies the same transformation to the pattern characters and uses the index to quickly determine a set of positions where the pattern may occur. To discard false positives fm-oppm does a final verification step using the original text.

Finally, we compare smi with the algorithm simd-oppm [3]. simd-oppm does not use an index so it finds order-preserving matchings by testing every text position. However, to verify the matching simd-oppm exploits in a clever way the SIMD parallelism offered by the SSE instruction set; as a result simd-oppm is in practice the fastest among the order-preserving matching algorithms that do not use an index [3, Section 4].

We tested all algorithms using the collection of files listed in Fig. 1 1 which includes real and synthetic data. The test files, as well as the source code of all tested algorithms, are available at the repository https://gitlab.com/manzai/order-preserving-index. All 
tests have been performed on a desktop PC with eight Intel-I7 3.40GHz CPUs running Linux-Debian 8.3 using the gcc compiler version 4.9.2 and optimization option -03. All tests used a single CPU while the PC was not performing any other significant computation.

\subsection{Space usage}

Table 1 shows the space usage of our index for different values of $B$ and $q$ compared to the compression tools gzip and $x z$. We can see that smi's space usage is essentially at par with gzip's: it can be smaller or larger depending on the block size $B$. As expected $x z$ compression is clearly superior to both. Table 2 shows the relative space usage, within smi, of the compressed suffic array (csa) for $T_{o}$ vs. the long-skewed encoding (Isk) of $T_{\delta}$. For a fixed block size $B$, as the window size $q$ increases, the cost of storing $T_{\delta}$ decreases while the csa size increases. This was to be expected since a larger $q$ means that more information is contained in $T_{o}$. For a fixed window size $q$, as the block size $B$ increases, the space of both $T_{\delta}$ and $T_{o}$ 's csa decreases since both structures have an extra overhead for each block. However, increasing the block size decreases the search speed as discussed in the following section.

\subsection{Search time}

All search tests involved 1000 patterns of length 10, 15 and 20 extracted from the same file where the patterns are later sought, so every search reports at least one occurrence. The patterns were extracted selecting 1000 random positions in the file. Note that patterns occurring more often are more likely to be selected so this setting is the least favorable for our algorithm: like all index-based algorithms, it is much faster when the pattern does not occur, or occurs relatively few times.

Since Phases 1 and 2 of our algorithm produce a set of candidates that must be verified in Phase 3, in our first experiment we measure how effective are the first two Phases in producing only a small number of candidates which are later discarded (that is, how effective are Phases 1 and 2 in producing a small number of false positives). The results of this experiment are reported in Table 3. Note that the number of false positives does not depend on the block size $B$ that only affects the space usage and running time.

We see that for patterns of length 10 the number of false positives can be very high especially for $q=3$ and $q=9$. This is due to two different phenomena. For $q=3$, the order component $T_{o}$ has the least amount of information on the actual ordering, so even after Phase 2 the number of false positives is significant. For $q=9, T_{o}$ is much more informative but during Phase 1 we search in $T_{o}$ csa only the two symbols $P_{o}[9,10]$ (see Section 3.3) and therefore we are left with a large number of candidates. In Phase 2 however, we make use of $P_{o}[2,8]$ and the number of false positives drops below those of the case $q=3$.

As the size of the pattern increases, we see that the number of false positives decreases significantly. For $|P|=20$ at the end of Phase 2 the number of false positives is at most $64 \%$ of the number of occurrences for $q=3$ and such percentage drops to $5 \%$ for $q=6$ and $1 \%$ for $q=9$. The bottom line is that the number of false positives appears to be reasonably small except in extreme cases when $q$ is very small or when $q$ is very close to the length of the pattern. Note also that there is a large variability in the number of false positives across the different input files. 


\begin{tabular}{|l|l|r|r|r|r|r|r|}
\hline & prices & temp & ecg & rwalk & rand & ran127 \\
\hline \hline \multirow{3}{*}{$B=32$} & smi $q=3$ & 0.35 & 0.31 & 0.27 & 0.34 & 0.35 & 0.43 \\
\cline { 2 - 8 } & smi $q=6$ & 0.37 & 0.33 & 0.29 & 0.35 & 0.35 & 0.43 \\
\cline { 2 - 8 }$B=64$ & smi $q=9$ & 0.38 & 0.34 & 0.30 & 0.37 & 0.36 & 0.44 \\
\cline { 2 - 8 } & smi $q=12$ & 0.39 & 0.35 & 0.31 & 0.38 & 0.37 & 0.45 \\
\hline \hline \multirow{5}{*}{$B=96$} & smi $q=3$ & 0.29 & 0.25 & 0.21 & 0.27 & 0.28 & 0.37 \\
\cline { 2 - 8 } & smi $q=6$ & 0.30 & 0.26 & 0.22 & 0.28 & 0.28 & 0.37 \\
\cline { 2 - 8 } & smi $q=9$ & 0.31 & 0.27 & 0.23 & 0.30 & 0.29 & 0.37 \\
\cline { 2 - 8 } & smi $q=12$ & 0.32 & 0.28 & 0.24 & 0.30 & 0.30 & 0.37 \\
\hline \hline \multirow{3}{*}{} & smi $q=3$ & 0.27 & 0.23 & 0.19 & 0.24 & 0.26 & 0.34 \\
\cline { 2 - 8 } & smi $q=6$ & 0.28 & 0.24 & 0.20 & 0.26 & 0.26 & 0.34 \\
\cline { 2 - 8 }$q=9$ & 0.29 & 0.25 & 0.21 & 0.27 & 0.27 & 0.35 \\
\cline { 2 - 8 } & smi $q=12$ & 0.30 & 0.26 & 0.22 & 0.28 & 0.27 & 0.35 \\
\hline \hline & gzip --best & 0.37 & 0.24 & 0.19 & 0.36 & 0.24 & 0.35 \\
\cline { 2 - 3 } & 0.24 & 0.17 & 0.12 & 0.23 & 0.18 & 0.29 \\
\hline \hline
\end{tabular}

Table 1: smi overall space usage for different values of $B$ and $q$. Each value is the ratio between the size of the index over the size of the input file, both expressed in bytes. Also shown are the space usage for gzip and xz.

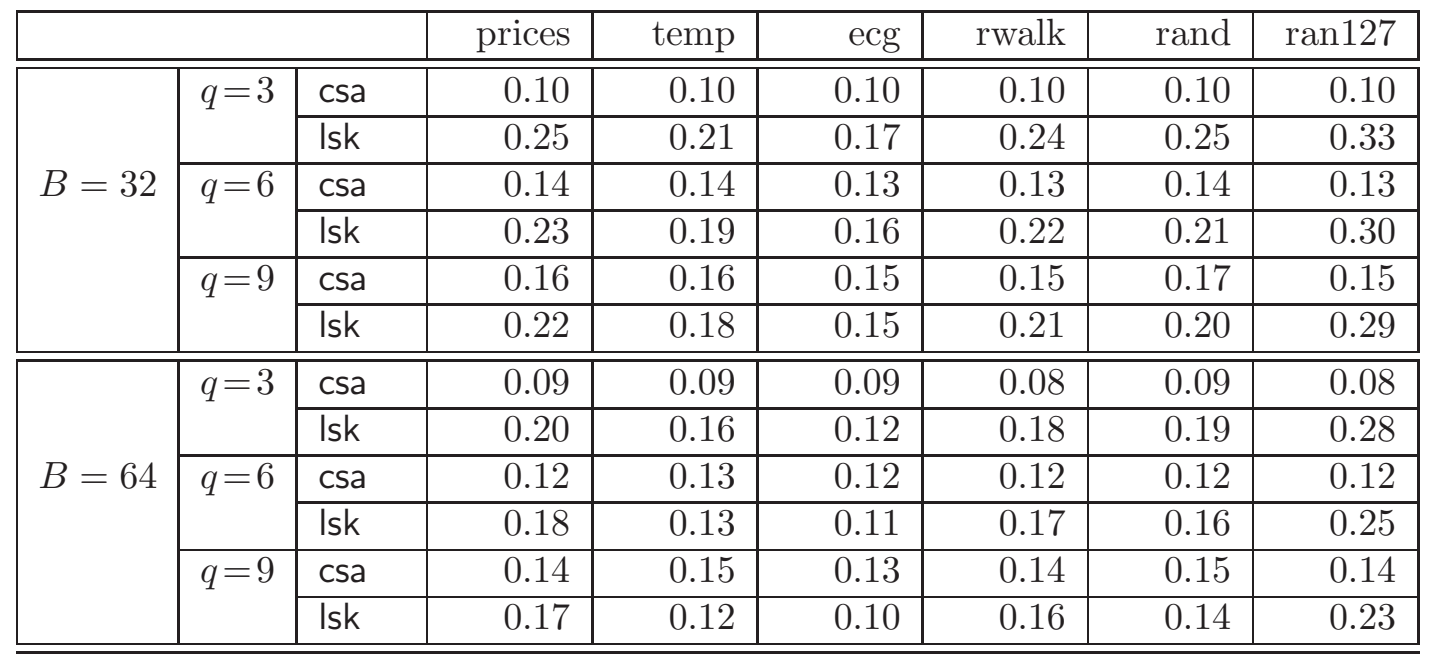

Table 2: Space usage of $T_{o}$ 's compressed suffix array (csa) and of $T_{\delta}$ 's log-skewed encoding (Isk) for different values of $B$ and $q$. The reported values are the ratio between the size of the csa/lsk file over the size of the input file both expressed in bytes. 
Table 4 reports the average running times of smi for different values of $q$ and $B$, compared with those of the naive algorithm scan and of the algorithm fm-oppm from [5] (see description at the beginning of Section 4). We see that our algorithm is the fastest for every instance and the difference is by at least an order of magnitude for the larger pattern lengths and $B=32$. If we look at the running times for pattern lengths 15 and 20 we see that, as expected for an index data structure, the running time grows linearly with the number of occurrences and does not depend on the size of the input. For example, the search is much faster for rand than for ecg since the latter is much shorter but has a much larger number of occurrences. For patterns of length 10 we see that the running time can be influenced by the presence of a large number of false positives at the end of Phase 2 . For example, the two files rwalk and ran127 have the same length; the former has a much higher number of occurrences, but the search times are relatively close since the latter has a much higher number of false positives. Finally, we observe that doubling the block size from $B=32$ to $B=64$ roughly doubles the search time for all values of $q$. Since the size of the block influences the running time only in Phase 3, when we decompress portions of $T_{\delta}$, this is an indirect confirmation that Phase 3 is indeed the most expensive of the algorithm. Since increasing the block size from $B=32$ to $B=64$ improves compression only slightly (see Table 2), the lesson we learn here is that larger blocks should be used only when space is at a premium.

The algorithm fm-oppm is also based on an index: before the search fm-oppm builds an FM-index on the binary string encoding whether the next character is smaller than the current one or not. Therefore, in some sense fm-oppm search operations are similar to the ones we would have for smi with $q=2$ (in our implementation we can only have $3 \leq q \leq 128$ so we could not test this). Note however that we also keep explicit track of equalities of text values, so with $q=3$ each entry in $T_{o}$ can assume five distinct values instead of two as in fm-oppm. For $|P|=10$ we see that fm-oppm's running times are between 4 and 14 times smi's corresponding running times for $q=3$ and $B=32$. For larger values of $|P|$ the gap between fm-oppm and smi running times increases. With some additional tests, not reported here, we found that the likely reason is that even for $|P|=20$ the search in fm-oppm's index still produces a large number of false positives that have to be checked and discarded.

As expected, scan running time is roughly proportional to the input file length and is scarcely affected by the pattern length and the number of occurrences. Note that for $P=15$ and $P=20$ scan is at least two orders of magnitude slower than smi for $B=32$, with the only exception of the input file ecg and $|P|=15$ which have an unusually high average number of occurrences (more than 2000).

In Table 5 we report a comparison including the algorithm simd-oppm [3], which is the fastest algorithm among those that do not use an index. The available version of simdoppm is optimized for small values so it only accepts input files with values in the range $[-127,127]$. In our datasets of Table 1 the files temp, rand and ran127 already satisfy this restriction. To include also the other files we changed them by transforming each value $x$ to $(x \bmod 255)-127$. With this transformation all values are forced into the range $[-127,127]$ and, if neighboring values are not too distant, their relative order is usually unchanged. In Table 5 the names of the files whose values has been modified with the above transformation are shown in boldface.

From the results in Table 5 we see that simd-oppm running time is proportional to the input file length and is roughly thirty times faster than scan. Indeed, for $|P|=10$ simd-oppm is the fastest algorithm for the (modified) ecg file and is faster than smi for 
$q=3$ also for randw, rand and ran127. However, for larger values of the pattern length smi becomes significantly faster than simd-oppm. We also notice that for $|P|=15$ there is not a clear winner between fm-oppm and simd-oppm, while for $|P|=20 \mathrm{fm}$-oppm is consistently faster.

Summing up, we believe that our experiments indicate that for sufficiently large files it pays to build an index also for the order-preserving matching problem. Compared to the exact matching problem, the index performance for order-preserving matching is less predictable because the proposed indices, smi and fm-oppm, are only "approximate" and must deal with the possible presence of false positives. Nevertheless, the advantages of using an index are clear, especially when the index can be compressed to use significantly less space than the original file, as in smi. On the other hand, carefully engineered scanbased algorithms, like simd-oppm, will always be needed for short-medium files, or for very short patterns, or for the case in which the text cannot be preprocessed in advance.

\section{Concluding Remarks}

In this paper we have proposed a compressed index for the order-preserving pattern matching problem. Our approach is based on the new idea of splitting the original sequence into two complementary components: the order component and the $\delta$ component. The problem of finding the order-preserving occurrences of a pattern is transformed into an exact search problem on the order component followed by a verification phase using the $\delta$ component. Experiments show that our index has a space usage similar to gzip and can find order-preserving occurrences much faster than a sequential scan.

Our approach is quite general and improvements could be obtained by changing some implementation choices. For example, we index the order component using a Wavelet-Tree based FM-index; to improve the performances for inputs with many (order-preserving) repetitions we can use a different compressed full-text index, for example adapting the one recently proposed in [16]. Notice also that the compression of the $\delta$ component can be radically changed without altering the overall scheme.

Finally, we define the order component considering the position of the predecessor of each element in a sliding window. It is natural to try to extend the approach by also considering the successor. This can be done representing each element with a 〈predecessor, successor $\rangle$ pair, or using a second index storing the successor information.

\section{Acknowledgments}

The authors would like to than Jorma Tarhio and Tamanna Chhabra for providing the code of simd-oppm and fm-oppm, and for assistance in the testing of those algorithms.

\section{References}

[1] Djamal Belazzougui, Adeline Pierrot, Mathieu Raffinot, and Stéphane Vialette. Single and multiple consecutive permutation motif search. In ISAAC, volume 8283 of Lecture Notes in Computer Science, pages 66-77. Springer, 2013.

[2] Domenico Cantone, Simone Faro, and M. Oguzhan Külekci. An efficient skip-search approach to the order-preserving pattern matching problem. In Stringology, pages 
22-35. Department of Theoretical Computer Science, Czech Technical University in Prague, 2015.

[3] Tamanna Chhabra, Simone Faro, M. Oguzhan Külekci, and Jorma Tarhio. Engineering order-preserving pattern matching with SIMD parallelism. Softw., Pract. Exper., 47(5):731-739, 2017.

[4] Tamanna Chhabra, Emanuele Giaquinta, and Jorma Tarhio. Filtration algorithms for approximate order-preserving matching. In SPIRE, volume 9309 of Lecture Notes in Computer Science, pages 177-187. Springer, 2015.

[5] Tamanna Chhabra, M. Oguzhan Külekci, and Jorma Tarhio. Alternative algorithms for order-preserving matching. In Stringology, pages 36-46. Department of Theoretical Computer Science, Czech Technical University in Prague, 2015.

[6] Tamanna Chhabra and Jorma Tarhio. Order-preserving matching with filtration. In SEA, volume 8504 of Lecture Notes in Computer Science, pages 307-314. Springer, 2014 .

[7] Tamanna Chhabra and Jorma Tarhio. A filtration method for order-preserving matching. Inf. Process. Lett., 116(2):71-74, 2016.

[8] Sukhyeun Cho, Joong Chae Na, Kunsoo Park, and Jeong Seop Sim. A fast algorithm for order-preserving pattern matching. Inf. Process. Lett., 115(2):397-402, 2015.

[9] Maxime Crochemore, Costas Iliopoulos, Tomasz Kociumaka, Marcin Kubica, Alessio Langiu, Solon Pissis, Jakub Radoszewski, Wojciech Rytter, and Tomasz Walen. Order-preserving incomplete suffix trees and order-preserving indexes. In SPIRE, volume 8214 of Lecture Notes in Computer Science, pages 84-95. Springer, 2013.

[10] Maxime Crochemore, Costas S. Iliopoulos, Tomasz Kociumaka, Marcin Kubica, Alessio Langiu, Solon P. Pissis, Jakub Radoszewski, Wojciech Rytter, and Tomasz Walen. Order-preserving indexing. Theor. Comput. Sci., 638:122-135, 2016.

[11] Gianni Decaroli, Travis Gagie, and Giovanni Manzini. A compact index for orderpreserving pattern matching. In $D C C$, pages 72-81. IEEE, 2017.

[12] Simone Faro and M. Oguzhan Külekci. Efficient algorithms for the order preserving pattern matching problem. In AAIM, volume 9778 of Lecture Notes in Computer Science, pages 185-196. Springer, 2016.

[13] Paolo Ferragina and Giovanni Manzini. An experimental study of a compressed index. Inf. Sci., 135(1-2):13-28, 2001.

[14] Paolo Ferragina and Giovanni Manzini. Indexing compressed text. Journal of the ACM (JACM), 52(4):552-581, 2005.

[15] Travis Gagie, Giovanni Manzini, and Rossano Venturini. An encoding for orderpreserving matching. In ESA, volume 87 of LIPIcs, pages 38:1-38:15. Schloss Dagstuhl - Leibniz-Zentrum fuer Informatik, 2017.

[16] Travis Gagie, Gonzalo Navarro, and Nicola Prezza. Optimal-time text indexing in bwt-runs bounded space. In SODA, pages 1459-1477. SIAM, 2018. 
[17] Pawel Gawrychowski and Przemyslaw Uznanski. Order-preserving pattern matching with $k$ mismatches. In CPM, volume 8486 of Lecture Notes in Computer Science, pages 130-139. Springer, 2014.

[18] Simon Gog, Timo Beller, Alistair Moffat, and Matthias Petri. From theory to practice: Plug and play with succinct data structures. In SEA, volume 8504 of Lecture Notes in Computer Science, pages 326-337. Springer, 2014.

[19] D. Gusfield. Algorithms on Strings, Trees, and Sequences: Computer Science and Computational Biology. Cambridge University Press, 1997.

[20] Jinil Kim, Peter Eades, Rudolf Fleischer, Seok-Hee Hong, Costas S. Iliopoulos, Kunsoo Park, Simon J. Puglisi, and Takeshi Tokuyama. Order-preserving matching. Theor. Comput. Sci., 525:68-79, 2014.

[21] Marcin Kubica, Tomasz Kulczynski, Jakub Radoszewski, Wojciech Rytter, and Tomasz Walen. A linear time algorithm for consecutive permutation pattern matching. Inf. Process. Lett., 113(12):430-433, 2013.

[22] Giovanni Manzini and Marcella Rastero. A simple and fast DNA compressor. Softw., Pract. Exper., 34(14):1397-1411, 2004.

[23] Gonzalo Navarro and Veli Mäkinen. Compressed full-text indexes. ACM Comput. Surv., 39(1), 2007. 


\begin{tabular}{|l|r|r|r|r|r|r|r|}
\hline$|P|=10$ & prices & temp & ecg & rwalk & rand & ran127 \\
\hline \hline \multicolumn{2}{|l|}{ ave \# occs } & 431.45 & 8.42 & 19801.90 & 802.39 & 3.03 & 10.66 \\
\hline \hline \multirow{2}{*}{$q=3$} & Phase 1 & 8.02 & 311.89 & 1.76 & 12.81 & 2984.41 & 1470.69 \\
\cline { 2 - 8 } & Phase 2 & 4.81 & 182.44 & 1.19 & 8.13 & 2035.82 & 1033.44 \\
\hline \hline$q=6$ & Phase 1 & 26.31 & 274.70 & 5.47 & 35.30 & 808.16 & 563.43 \\
\cline { 2 - 8 } & Phase 2 & 1.83 & 10.11 & 1.04 & 2.81 & 53.33 & 53.21 \\
\hline \hline$q=9$ & Phase 1 & 1193.64 & 30119.77 & 42.96 & 1325.32 & 104819.21 & 50448.11 \\
\cline { 2 - 8 } & Phase 2 & 3.72 & 15.79 & 1.05 & 6.06 & 221.74 & 308.66 \\
\hline
\end{tabular}

\begin{tabular}{|c|c|c|c|c|c|c|c|}
\hline \multicolumn{2}{|c|}{$|P|=15$} & prices & temp & ecg & rwalk & rand & $\operatorname{ran} 127$ \\
\hline \multicolumn{2}{|c|}{ ave \# occs } & 59.70 & 1.00 & 2015.25 & 2.38 & 1.00 & 1.00 \\
\hline \multirow[t]{2}{*}{$q=3$} & Phase 1 & 1.37 & 10.10 & 2.01 & 23.62 & 49.70 & 119.48 \\
\hline & $\overline{\text { Phase } 2}$ & 1.16 & 6.64 & 1.23 & 15.36 & 34.53 & 85.26 \\
\hline \multirow[t]{2}{*}{$q=6$} & Phase 1 & 3.31 & 1.47 & 4.48 & 20.26 & 1.20 & 2.25 \\
\hline & $\overline{\text { Phase } 2}$ & 1.02 & 1.02 & 1.03 & 2.21 & 1.01 & 1.11 \\
\hline \multirow[t]{2}{*}{$q=9$} & Phase 1 & 11.04 & 5.39 & 13.03 & 438.92 & 2.11 & 9.19 \\
\hline & $\overline{\text { Phase } 2}$ & 1.04 & 1.00 & 1.03 & 3.24 & 1.00 & 1.06 \\
\hline
\end{tabular}

\begin{tabular}{|l|r|r|r|r|r|r|r|}
\hline$|P|=20$ & prices & temp & ecg & rwalk & rand & ran127 \\
\hline \hline \multicolumn{2}{|l|}{ ave \# occs } & 1.49 & 1.00 & 147.80 & 1.00 & 1.00 & 1.00 \\
\hline \hline$q=3$ & Phase 1 & 1.20 & 1.05 & 2.60 & 1.39 & 1.27 & 1.89 \\
\cline { 2 - 8 } & Phase 2 & 1.12 & 1.03 & 1.42 & 1.29 & 1.17 & 1.64 \\
\hline \hline$q=6$ & Phase 1 & 2.49 & 1.00 & 5.34 & 1.08 & 1.00 & 1.00 \\
\cline { 2 - 8 } & Phase 2 & 1.00 & 1.00 & 1.05 & 1.02 & 1.00 & 1.00 \\
\hline \hline$q=9$ & Phase 1 & 47.19 & 1.00 & 17.17 & 2.10 & 1.00 & 1.00 \\
\cline { 2 - 8 } & Phase 2 & 1.01 & 1.00 & 1.02 & 1.01 & 1.00 & 1.00 \\
\hline
\end{tabular}

Table 3: False positives as a function of the window size $q$ for 1000 patterns of length 10, 15 and 20. The first row shows the average number of actual occurrences for the patterns in the test set. The other rows show the ratios between candidates and actual occurrences at the end of Phase 1 and 2, obtained as the sum of candidates over all patterns over the sum of actual occurrences over all patterns. 


\begin{tabular}{|l|r|r|r|r|r|r|r|}
\hline$|P|=10$ & prices & temp & ecg & rwalk & rand & ran127 \\
\hline \hline \multicolumn{2}{|l|}{ ave \# occ } & 431.45 & 8.42 & 19801.90 & 802.39 & 3.03 & 10.66 \\
\hline \hline \multirow{3}{*}{$B=32$} & smi $q=3$ & 7.52 & 6.16 & 64.82 & 27.17 & 26.96 & 46.27 \\
\cline { 2 - 8 } & smi $q=6$ & 4.15 & 0.62 & 75.82 & 15.01 & 1.35 & 4.28 \\
\cline { 2 - 8 } & smi $q=9$ & 13.81 & 2.13 & 91.06 & 47.47 & 10.55 & 40.20 \\
\hline \hline \multirow{3}{*}{$B=64$} & smi $q=3$ & 13.68 & 10.89 & 125.11 & 50.37 & 50.27 & 87.06 \\
\cline { 2 - 8 } & smi $q=6$ & 7.59 & 1.09 & 151.87 & 28.12 & 2.40 & 7.75 \\
\cline { 2 - 8 } & smi $q=9$ & 23.40 & 3.07 & 182.84 & 83.77 & 16.16 & 65.70 \\
\hline \hline fm-oppm & 70.42 & 74.74 & 256.85 & 185.07 & 399.92 & 398.79 \\
\hline \hline \multicolumn{2}{|l|}{ scan } & 246.19 & 239.98 & 134.51 & 408.60 & 420.18 & 442.69 \\
\hline
\end{tabular}

\begin{tabular}{|c|c|c|c|c|c|c|c|}
\hline \multicolumn{2}{|c|}{$|P|=15$} & prices & temp & ecg & rwalk & rand & ran127 \\
\hline \multicolumn{2}{|c|}{ ave \# occ } & 59.70 & 1.00 & 2015.25 & 2.38 & 1.00 & 1.00 \\
\hline \multirow{3}{*}{$B=32$} & smi $q=3$ & 0.19 & 0.03 & 6.96 & 0.17 & 0.17 & 0.40 \\
\hline & smi $q=6$ & 0.19 & 0.02 & 7.58 & 0.05 & 0.02 & 0.02 \\
\hline & smi $q=9$ & 0.21 & 0.02 & 8.83 & 0.13 & 0.03 & 0.03 \\
\hline \multirow{3}{*}{$B=64$} & smi $q=3$ & 0.35 & 0.06 & 13.28 & 0.30 & 0.30 & 0.71 \\
\hline & smi $q=6$ & 0.37 & 0.02 & 15.13 & 0.08 & 0.02 & 0.03 \\
\hline & smi $q=9$ & 0.39 & 0.03 & 17.90 & 0.19 & 0.04 & 0.04 \\
\hline \multicolumn{2}{|c|}{ fm-oppm } & 2.52 & 2.60 & 50.82 & 6.18 & 21.05 & 21.74 \\
\hline \multicolumn{2}{|l|}{ scan } & 248.71 & 240.32 & 134.43 & 403.49 & 406.74 & 454.96 \\
\hline
\end{tabular}

\begin{tabular}{|l|r|r|r|r|r|r|r|}
\hline$|P|=20$ & prices & temp & ecg & rwalk & rand & ran127 \\
\hline \hline \multicolumn{2}{|l|}{ ave \# occ } & 1.49 & 1.00 & 147.80 & 1.00 & 1.00 & 1.00 \\
\hline \hline \multirow{3}{*}{$B=32$} & smi $q=3$ & 0.01 & 0.01 & 0.66 & 0.01 & 0.01 & 0.01 \\
\cline { 2 - 8 } & smi $q=6$ & 0.02 & 0.02 & 0.63 & 0.02 & 0.02 & 0.02 \\
\cline { 2 - 8 } & smi $q=9$ & 0.03 & 0.03 & 0.74 & 0.03 & 0.03 & 0.03 \\
\hline \hline \multirow{3}{*}{$B=64$} & smi $q=3$ & 0.02 & 0.01 & 1.16 & 0.02 & 0.02 & 0.02 \\
\cline { 2 - 8 } & smi $q=6$ & 0.03 & 0.03 & 1.19 & 0.03 & 0.03 & 0.03 \\
\cline { 2 - 8 } & smi $q=9$ & 0.04 & 0.03 & 1.41 & 0.04 & 0.04 & 0.04 \\
\hline \hline fm-oppm & 0.12 & 0.13 & 8.86 & 0.23 & 1.18 & 1.31 \\
\hline \hline \multicolumn{2}{|l|}{ scan } & 241.21 & 234.43 & 133.22 & 402.90 & 405.34 & 468.98 \\
\hline
\end{tabular}

Table 4: Average smi running times, in milliseconds, for searching 1000 random patterns of length 10, 15, and 20 for different values of $B$ and $q$ compared with the running times of the algorithms scan and fm-oppm. Running times do not include the time to load/compute the compressed index (for smi and fm-oppm) or the uncompressed text (for scan and fm-oppm). 


\begin{tabular}{|l|r|r|r|r|r|r|}
\hline$|P|=10$ & prices & temp & ecg & rwalk & rand & ran127 \\
\hline \hline ave \# occ & 358.22 & 8.42 & 7460.50 & 622.32 & 3.03 & 10.66 \\
\hline \hline smi $q=3$ & 6.37 & 6.16 & 27.76 & 22.42 & 26.96 & 46.27 \\
\hline smi $q=6$ & 2.19 & 0.62 & 31.73 & 10.97 & 1.35 & 4.28 \\
\hline smi $q=9$ & 9.30 & 2.13 & 40.09 & 42.38 & 10.55 & 40.20 \\
\hline \hline simd-oppm & 7.41 & 6.70 & 5.36 & 14.14 & 11.81 & 14.08 \\
\hline \hline fm-oppm & 74.76 & 71.88 & 133.73 & 187.01 & 397.84 & 397.93 \\
\hline \hline scan & 254.01 & 239.98 & 139.96 & 417.25 & 420.18 & 442.69 \\
\hline
\end{tabular}

\begin{tabular}{|l|r|r|r|r|r|r|}
\hline$|P|=15$ & prices & temp & ecg & rwalk & rand & ran127 \\
\hline \hline ave \# occ & 169.93 & 1.00 & 880.49 & 1.22 & 1.00 & 1.00 \\
\hline \hline smi $q=3$ & 0.42 & 0.03 & 3.17 & 0.12 & 0.17 & 0.40 \\
\hline smi $q=6$ & 0.45 & 0.02 & 3.79 & 0.03 & 0.02 & 0.02 \\
\hline smi $q=9$ & 0.50 & 0.02 & 4.47 & 0.07 & 0.03 & 0.03 \\
\hline \hline simd-oppm & 7.37 & 6.41 & 5.28 & 13.58 & 10.92 & 13.82 \\
\hline \hline fm-oppm & 2.93 & 2.57 & 17.05 & 6.47 & 21.00 & 21.63 \\
\hline \hline scan & 249.15 & 240.32 & 139.32 & 415.13 & 406.74 & 454.96 \\
\hline
\end{tabular}

\begin{tabular}{|l|r|r|r|r|r|r|}
\hline$|P|=20$ & prices & temp & ecg & rwalk & rand & ran127 \\
\hline \hline ave \# occ & 1.08 & 1.00 & 42.50 & 1.00 & 1.00 & 1.00 \\
\hline \hline smi $q=3$ & 0.01 & 0.01 & 0.22 & 0.01 & 0.01 & 0.01 \\
\hline smi $q=6$ & 0.02 & 0.02 & 0.21 & 0.02 & 0.02 & 0.02 \\
\hline smi $q=9$ & 0.03 & 0.03 & 0.26 & 0.03 & 0.03 & 0.03 \\
\hline \hline simd-oppm & 7.07 & 6.36 & 5.18 & 12.82 & 10.34 & 13.47 \\
\hline \hline fm-oppm & 0.15 & 0.12 & 2.20 & 0.26 & 1.26 & 1.38 \\
\hline \hline scan & 247.43 & 234.43 & 137.68 & 402.10 & 405.34 & 468.98 \\
\hline
\end{tabular}

Table 5: Average smi running times for searching 1000 random patterns of length 10, 15 , and 20 for different values of $q$ and $B=32$ compared with the running times of the algorithms simd-oppm, fm-oppm, and scan. Running times are in milliseconds and do not include the time to load/compute the compressed index (for smi and fm-oppm) or the uncompressed text (for simd-oppm, fm-oppm, and scan). The names of the files whose content has been modified to force all values in the range $[-127,127]$ is in boldface. 